\title{
Société Française de Virologie : où en sommes-nous deux ans après ?
}

\author{
Aure Saulnier ${ }^{1}$ \\ David Gilmer $^{2}$ \\ Noel Tordo 3,4 \\ ${ }^{1}$ À titre personnel \\ $<$ Aure.Saulnier@sanofi.com> \\ 2 Université de Strasbourg, CNRS, \\ IBMP UPR 2357, \\ 67000 Strasbourg, France \\ <david.gilmer@ibmp-cnrs.unistra.fr> \\ ${ }^{3}$ Institut Pasteur de Guinée, \\ Université Gamal Abdel Nasser, \\ Conakry, Guinée \\ ${ }^{4}$ Institut Pasteur, 25 rue du Dr. Roux, \\ 75724 Paris cedex 15, France \\ <ntordo@pasteur.fr>
}

\begin{abstract}
Chers Amis,
Voilà bientôt trois ans que nous avons créé ensemble la « Société Française de Virologie » $(\mathrm{SFV})^{1}$ qui correspond à une envie de meilleure visibilité partagée depuis longtemps par de nombreux virologistes et virologues. Nous ne choisissons volontairement pas entre ces deux termes dont l'usage courant montre la diversité et donc la richesse de notre communauté.
\end{abstract}

La mise en route de notre Société a été progressive mais rappelons ici les progrès enregistrés :

- Les «Journées Francophones de Virologie » (JFV) sont le congrès annuel de la SFV avec une promotion réciproque : un tarif d'inscription plus attrayant pour le couple «JFV-SFV » que pour «JFV » seul (voir le site https://jfv-2019.sciencesconf.org/). Ainsi, tous les participants aux JFV peuvent choisir (à moindre prix) de devenir membres de la SFV pour un an... ce qui n'empêche pas ceux qui ne peuvent participer aux JFV de régler leur cotisation annuelle directement à la SFV.

- Virologie est le journal de la SFV avec, là encore, une promotion réciproque : un tarif d'abonnement préférentiel pour les membres de la SFV. Nous bénéficions du partenariat constant que nous maintenons avec notre éditeur John Libbey Eurotext depuis la création de Virologie, il y a plus de 20 ans.

- Pour améliorer notre visibilité, nous sommes en train de finaliser le site internet de la SFV. Il sera fonctionnel dans quelques mois et il faudra alors le faire vivre : nous comptons sur vous, notamment sur les « geeks », pour le nourrir d'informations fraîches et attrayantes.

- Déjà, la SFV est visible à travers la page du réseau professionnel LinkedIn que nous avons créée. Aure Saulnier et David Gilmer sont les animatrice(eur)s de cette page où des informations régulières circulent déjà, qu'ils en soient vivement remerciés.

- La SFV a commencé à soutenir des conférences/congrès/workshops, traitant de virologie, et organisé(e)s sur le territoire national. Elle aide ainsi des doctorant(e)s (notamment en fin de thèse) dont le laboratoire/directrice(eur) est membre de la $\mathrm{SFV}$, à présenter leurs résultats (oral, poster) et à nouer des connexions futures durant différents congrès nationaux et internationaux. Les demandes sont déposées via le site JFV (« onglet bourses ») ou envoyées par courriel à la SFV en cas de dysfonctionnement du site. Elles sont étudiées deux fois par an (janvier, juin) lors des réunions du conseil d'administration (CA). Les lauréats s'engagent à faire apparaître le logo de la SFV sur leur site web/diaporama/poster et à rédiger un compte rendu (environ une page A4) qui sera relu puis publié dans Virologie.

- La SFV reste ouverte à l'interaction avec d'autres sociétés nationales et internationales. Elle est membre de la Société Européenne de Virologie à l'instar

\footnotetext{
${ }^{1}$ Voir Le Comité d'organisation des JFV. Création de la Société Française de Virologie en 2015 : la fonction crée l'organe. Virologie 2015 ; 19 : 41-2. DOI : 10.1684/vir.2015.0604.
} 


\section{éditorial}

de la plupart des autres sociétés nationales européennes. Elle a établi un partenariat avec la Société Française de Microbiologie (SFM) qui se traduit notamment par :

- la participation d'un invité permanent de la SFM au CA de la SFV et réciproquement ;

- l'organisation d'une « session virologie » par la SFV durant le congrès de la SFM et l'organisation par la SFM d'une session « interaction bactéries/parasites-virus » durant les JFV ;

- l'édition, supportée par la SFM, d'une mise à jour du Traité de virologie médicale ${ }^{2}$ auquel participent plusieurs membres de la SFV. Les bénéfices éventuels, au-delà du remboursement des frais d'édition, seront partagés entre les deux sociétés ;

- la participation commune SFV/SFM (06/09/2018) à une réunion des responsables de 49 sociétés savantes académiques et d'associations/institutions à l'interface entre le monde académique et la société. Cette réunion avait pour objectif d'initier une réflexion commune sur les missions et modes d'organisation des sociétés et de faire émerger des propositions concrètes d'actions futures collectives pour faire mieux entendre la voix du monde académique dans le débat public et pour une meilleure prise en compte des apports de la science dans le processus de décision politique.

L'ensemble de ces activités (figure 1) ne représente qu'une mise en route. Comme chaque société débutante et même si « l'envie de SFV » était ancrée depuis longtemps en France, nous cherchons à optimiser nos actions et sommes évidemment à votre écoute pour les améliorer car la SFV n'est rien d'autre que « notre société » : elle est et sera à l'image que ce que nous voulons/voudrons en faire.

Je finis en solo en tant que Président de la SFV pour écorcher la modestie de ma cosignataire et responsable actuelle du Secrétariat de la SFV, Aure Saulnier, qui a réalisé le poster ci-dessous. Il résume de façon synoptique et bilingue à celles et ceux qui ne la connaîtraient pas ce qu'est la SFV, son organisation, ses missions et ses organes de diffusion.

Bien amicalement à toutes et tous,

Noël Tordo

Liens d'intérêts : les auteurs déclarent ne pas avoir de lien d'intérêt en rapport avec cet éditorial.

\footnotetext{
${ }^{2}$ Hureaux JM, Nicolas JC, Agut H, Peigue-Lafeuille H. Traité de virologie médicale. Paris : éditions Estem, 2003.
} 


\section{Objectif}

L'objectif de la Société Française de Virologie (SFV) est la promotion et le soutien de la virologie sous ses aspects de recherche, de formation et autres applications.

\section{Organisation}

La société savante SFV est une association placée sous le régime de la loi du $1^{\text {er }}$ juillet 1901 . Crée en 2016, l'association se compose de membres adhérents (cotisation annuelle potentiellement couplée à l'inscription aux " Journées Francophones de Virologie ", JFV). Elle est administrée par un Conseil d'Administration (CA) composé de 10 membres élus par bulletin secret pour 4 ans. Le Bureau du CA comprend 1 Président, 1 Trésorier, 1 Trésorier Adjoint, 1 Vice-Président, 1 Secrétaire, 1 Secrétaire-Adjoint. L'Assemblée Générale de la Société a lieu tous les ans durant le congrès annuel (JFV). La SFV a le souci de maintenir un lien étroit avec la Société Française de Microbiologie.

\section{Missions}

La SFV poursuit notamment les buts suivants :

- Elle promeut les échanges d'information et la collaboration entre ses membres ainsi qu'avec les autres Sociétés savantes Françaises, Européennes, ou Internationales.

- Elle a pour objectif d'obtenir les moyens financiers correspondant à ses missions.

- Elle apporte son soutien aux scientifiques et praticiens travaillant dans le domaine de la virologie.

- Elle est un interlocuteur avertides pouvoirs publics en matière de recherche fondamentale, appliquée et clinique, de prévention et d'anticipation des crises sanitaires ou environnementales touchant l'ensemble du vivant soumis à une menace de nature virologique.

- Elle est un relais vers les médias et le public.

\section{Organes de diffusion}

- "Virologie" http://www.jle.com/en/revues/vir/revue.phtml est la revue officielle de la SFV et les " Journées Francophones de Virologie " constituent son congrès annuel, rassemblant des virologistes des domaines public/privé, médecins, biologistes, vétérinaires.

- La gestion du congrès annuel se fait via le site https://ifv2019.sciencesconf.org/. Un site dédié est en cours de construction. Une page du réseau professionnel Linkedln permet également de mettre en lien des virologistes de différents horizons, français ou étrangers, et de répondre aux missions de la SFV.

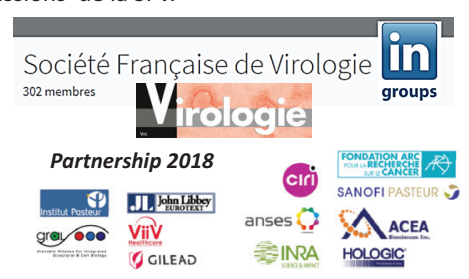

\begin{tabular}{|c|c|c|}
\hline \multicolumn{3}{|c|}{ XX Journées francophones de Virologie - 2018.03.29-30 } \\
\hline participants & 450 & attendees \\
\hline présentations scientifiques & 167 & scientific communications \\
\hline plénières - orateurs invités & 18 & plenary sessions - invited speakers \\
\hline communications orales & 44 & oral presentations \\
\hline communications affichées & 105 & posters \\
\hline communication transversale - Bioaster & 1 & transversal sciences - Bioaster presentation \\
\hline \multicolumn{3}{|c|}{2018 : Financement bourses \& congrès - Sponsoring \& Funding } \\
\hline $\begin{array}{r}\text { bourses d'étudiant pour participation } \\
\text { à un congrès international }\end{array}$ & 2 & $\begin{array}{l}\text { international congress fellowship for } \\
\text { student }\end{array}$ \\
\hline congrès sponsorisé & 1 & congress sponsoring \\
\hline
\end{tabular}

Figure 1. La Société Française de Virologie (SFV) : son organisation, ses missions et ses organes de diffusion / The French Society for Virology: organization, missions and broadcasting media.

Goal

The goal oft he French Society for Virology "Société Française de Virologie" (SFV) is to promote and to support research, training and education in the field of virology.

\section{Organization}

The French Society for Virology (SFV) is a non-profit organization founded in 2016 (July 1st, 1901 French law for association), is composed off ull members (annual membership subscription possibly included in registration for "Journées Francophones de Virologie", JFV). The SFV is administered by an Executive Board composed of 10 members elected by secret ballotf or 4 years. The Executive Board Bureau consists of 1 President, 1 Treasurer, 1 Assistant Treasurer, 1 Vice-President, 1 Secretary, 1 Assistant Secretary. The SFV Ordinary General Meeting takes place every year during the annual congress (JFV). An important stated goal oft he SFV is to keep close interaction with the French Society off or Microbiology.

\section{Missions}

The main objectives of the SFV:

- To foster information sharing and collaboration between its members as well as with other French, European, and International learned Societies.

- To gather the financial means necessary to fulfil its missions.

- To support scientists and practitioners working in the field of virology.

- To be the privileged contactf or public authorities when it comes to fundamental, applied and clinical research, prevention and anticipation ofh ealth or environmental crises concerning all living organism facing a virus threat .

- To relay information and communication towards the medias and the public.

\section{Broadcasting media}

- "Virologie" http://www.jle.com/en/revues/vir/revue.phtml is the SFV journal and its annual congress, the "Journées Francophones de Virologie" gathers virologists from academia and industry, clinicians, biologists, veterinarians ...

- The annual congress is managed via the website https://ifv2019.sciencesconf.org/. A dedicated site is under construction. A SFV group on the Linkedln professional network helps to connect virologists all over the world, and to fulfil some oft he SFV missions. 